\title{
Reduced blood pressure after smooth muscle EFNB2 deletion and the potential association of EFNB2 mutation with human hypertension risk
}

\author{
Yujia Wang ${ }^{1,8}$, Pavel Hamet ${ }^{1,8}$, Eric Thorin ${ }^{2}$, Johanne Tremblay ${ }^{1}$, John Raelson ${ }^{1,3}$, Zenghui Wu1 ${ }^{1}$, Hongyu Luo ${ }^{1}$, \\ Wei Jin ${ }^{1}$, Julie L Lavoie ${ }^{1,4}$, Junzheng Peng ${ }^{1}$, Francois-Christophe Marois-Blanchet ${ }^{1}$, Muhammad Ramzan Tahir ${ }^{1}$, \\ John Chalmers ${ }^{5}$, Mark Woodward ${ }^{5}$, Stephen Harrap ${ }^{6}$, Shijie Qi ${ }^{1}$, Charles Yibin $\mathrm{Li}^{1}$ and Jiangping $\mathrm{Wu}^{\star}{ }^{\star 1,7}$
}

Ephrin B2 (EFNB2) is a ligand for erythropoietin-producing hepatocellular kinases (EPH), the largest family of receptor tyrosine kinases. It has critical functions in many biological systems, but is not known to regulate blood pressure. We generated mice with a smooth muscle cell (SMC)-specific deletion of EFNB2 and investigated its roles in blood pressure regulation and vascular SMC (VSMC) contractility. Male Efnb2 knockout (KO) mice presented reduced blood pressure, whereas female KO mice had no such reduction. Both forward signaling from EFNB2 to EPHs and reverse signaling from EPHs to EFNB2 were involved in regulating VSMC contractility, with EPHB4 serving as a critical molecule for forward signaling, based on crosslinking studies. We also found that a region from aa 313 to aa 331 in the intracellular tail of EFNB2 was essential for reverse signaling regulating VSMC contractility, based on deletion mutation studies. In a human genetic study, we identified five SNPs in the 3' region of the EFNB2 gene, which were in linkage disequilibrium and were significantly associated with hypertension for male but not female subjects, consistent with our findings in mice. The coding (minor) alleles of these five SNPs were protective in males. We have thus discovered a previously unknown blood pressure-lowering mechanism mediated by EFNB2 and identified EFNB2 as a gene associated with hypertension risk in humans.

European Journal of Human Genetics (2016) 24, 1817-1825; doi:10.1038/ejhg.2016.105; published online 17 August 2016

\section{INTRODUCTION}

Erythropoietin-producing hepatocellular kinases (EPH) are the largest family of receptor tyrosine kinases. In the basis of sequence homology, they are divided into A and B subfamilies. ${ }^{1}$ Their ligands, called ephrins (EFNs), are also cell surface molecules. ${ }^{2}$ EFNs are also divided into A and B subfamilies, based on the way they anchor on the cell surface: the A subfamily anchors on the cell surface through glycophosphatidylinositol, and the B subfamily, through a transmembrane domain. ${ }^{2}$ The interactions between EPH kinases and EFNs are promiscuous, but EPHA kinases preferably interact with EFNA ligands, and EPHB kinases with EFNB ligands, which have three members, EFNB1, EFNB2 and EFNB3. ${ }^{2}$ Although EPH members and EFN members share homology with their respective members, each member has its distinct function in different cellular processes. ${ }^{3-7}$ In general, the $\mathrm{EPH}$ kinases interact with their EFN ligands on neighboring cells, because EPHs and EFNs are all cell surface molecules. ${ }^{2}$ These molecules could be cleaved from the cell surface by enzymes such as $\mathrm{ADAM} 10,{ }^{8,9}$ an unspecified matrix metalloproteinase, ${ }^{10}$ or $\gamma$-secretase; ${ }^{11}$ therefore, it is possible that the shed soluble fragments of EPH and EFN might be able to influence cells and tissues at a distance by blocking the interaction of EPHs and EFNs there.
EPH kinases and EFNs are expressed in a wide range of tissues and cells, and have vital roles in the development and function of different organs and systems. ${ }^{3,4,12-22}$ They are also vital in many biological processes; ${ }^{5,6,23,24}$ however, until our recent publications, there were no studies investigating the roles of these molecules in blood pressure (BP) regulation.

Recently, we reported novel observations that deletion of EFNB1 and its receptor EPHB6 leads to increased $\mathrm{BP} .{ }^{25,26}$ In an additional study, we demonstrated that EPHB4 deletion leads to reduced BP. ${ }^{27}$ As EFNB2 is the preferred ligand of EPHB4, we thus asked the question whether its deletion in vascular smooth muscle cells (VSMCs) will have a similar BP reduction effect. The results of our investigation are reported here.

\section{MATERIALS AND METHODS}

Generation of SMC-specific Efnb2 and Ephb4 gene knockout (KO) mice

We have previously reported on the generation of Efnb2 floxed mice. ${ }^{14}$ They were backcrossed with C57BL/6J for 10 generations and then mated with smooth muscle myosin heavy chain promoter-driven Cre transgenic mice (smMHC-Cre-IRES-eGFP) in the C57BL/6 background $^{12}$ to obtain SMC cellspecific Efnb2 gene KO mice. The ages of the KO and WT mice for the in vivo

\footnotetext{
${ }^{1}$ Research Centre, Centre Hospitalier de l'Université de Montréal (CHUM), Montreal, QC, Canada; ${ }^{2}$ Department of Surgery, Université de Montréal and Université Montreal Heart Institute, Montreal, QC, Canada; ${ }^{3}$ PGX-Services, Montreal, QC, Canada; ${ }^{4}$ Department of Kinesiology, University of Montreal, Montreal, QC, Canada; ${ }^{5}$ Department of Biostatistics, The George Institute for Global Health, University of Sydney, Sydney, NSW, Australia; ${ }^{6}$ Department of Physiology, University of Melbourne, Melbourne, VIC, Australia; ${ }^{7}$ Department of Nephrology, Centre Hospitalier de I'Université de Montréal (CHUM), Montreal, QC, Canada

*Correspondence: Dr J Wu, Department of Nephrology, Centre Hospitalier de I'Université de Montréal (CHUM), CRCHUM- R12-428, 900 rue St. Denis, Montreal, QC, Canada H2X OA9. Tel: +514 890 8000. ext. 25164; Fax: +514 412 7944; E-mail: jianping.wu@umontreal.ca

8These authors contributed equally to this work.

Received 27 November 2015; revised 4 June 2016; accepted 5 July 2016; published online 17 August 2016
} 
study were described in given experiments. Cells from mice at 3-6 months of age were used for in vitro studies.

In some experiments, VSMCs from smooth muscle-specific Ephb4 gene KO mice were used. The generation and characterization of these mice are described in our recent publication. ${ }^{27}$

Reverse transcription/quantitative-PCR (RT/qPCR), immunofluorescence microscopy, BP measurement by radiotelemetry, VSMC isolation, measurement of VSMC contractility, $\mathrm{Ca}^{++}$flux measurement, lentivirus preparation and infection. These methods and primers (Supplementary Table 1) used are described in Supplementary Materials.

\section{Human genetic study}

The patient cohort consisted of 3409 European, Australian, Canadian and New Zealander Caucasians (Supplementary Table 2) who had been ascertained to be suffering from type 2 diabetes and at high risk for macrovascular or microvascular diabetes complications and who were recruited for the ADVANCE (Action in Diabetes and Vascular Disease: Peterax and Diamicron-MR Controlled Evaluation) clinical study. ${ }^{28,29}$ Patients were divided into male and female groups, and genetic association studies for a hypertension phenotype were performed for male and female groups separately. A detailed explanation of the methodology of the association studies is presented in the Supplementary Materials.

\section{RESULTS}

\section{SMC-specific deletion of EFNB2 in mice}

The floxed Efnb2 mice ${ }^{14}$ in the C57BL/6J background were crossed with transgenic mice expressing smooth muscle myosin heavy chain promoter-driven Cre recombinase (also in the C57BL/6J background ${ }^{12}$ to achieve SMC-specific deletion of EFNB2. The deletion of Efnb2 at the mRNA level in vascular smooth muscles, but not in the spleen, heart, brain or liver, was confirmed by RT-qPCR (Figure 1a; Supplementary Figure 1). The deletion EFNB2 at the protein level in VSMCs was further confirmed by immunofluorescence (Figure 1b) and immunoblotting (Figure 1c). These mice with SMC-specific deletion of EFNB2 were called KO mice. There was no compensative upregulation of other EPHB and EFNB subfamily members in VSMCs after EFNB2 deletion (Supplementary Figure 2). The small artery structure of the $\mathrm{KO}$ mice was comparable to that of the wild type (WT) mice in terms of media thickness and lumen sizes (Supplementary Figure 3). The KO and WT VSMCs had similar proliferative rates in vitro culture (Supplementary Figure 4), and had similar levels of $\alpha$-actin expression, a SMC marker (Supplementary Figure 5). Although EFNB2 deletion was achieved by smooth muscle myosin heavy chain promoter-driven Cre, and is not specific to SMC in the vascular system, we did not notice gross morphological and functional abnormalities in the digestive system, respiratory system and reproductive system in the $\mathrm{KO}$ mice.

\section{BP phenotype of EFNB2 KO mice}

The BP of KO and WT mice was recorded every $2 \mathrm{~min}$ for a period of $24 \mathrm{~h}$. All the time points were analyzed with a mixed-effects linear model, using genotype, individual mouse, sex and time as qualitative factors (Supplementary Table 3), and the mean+SEM of the values are presented in Figure 2. Male EFNB2 KO mice presented reduced BP (systolic pressure (SP), diastolic pressure (DP) and mean arterial pressure (MAP)) in general (the values of the WT parameters minus that of the $\mathrm{KO}$ were positive in Figure 2c), compared with their WT counterparts, with SP and MAP differences (WT vs KO) reaching significance (Figure 2a). Female KO mice, on the other hand, presented slightly increased $\mathrm{BP}$ parameters (the values of the difference were negative in Figure 2c) compared with their WT counterparts, with DP difference reaching significance (Figure $2 \mathrm{~b}$ ). The disparity (means + SEM of WT values minus KO values) of BP parameters between WT and KO mice are shown in Figure 2c. The males and females were significantly different for the disparity of DP and MAP (Figure 2c). The heart rates (HR) between WT and KO mice were not significantly different, and there was no sex differences (Figure 2d).

Both forward and reverse signaling between EFNB2 and its receptors are responsible for regulating VSMC contractility EFNB2 and its receptors (mainly EPHB subfamily kinases) are all expressed in VSMCs (Supplementary Figure 2), ${ }^{25}$ providing a molecular basis for their bi-directional signaling, ie, from EFNB2 to EPHBs: forward signaling; from EPHBs to EFNB2: reverse signaling. We investigated which direction was functional in regulating VSMC contractility. We demonstrated that when WT VSMCs were cultured in wells coated with recombinant EFNB2-Fc tagged with human IgG Fc (EFNB2-Fc), which could crosslink EPHBs and initiate forward signaling, they manifested significantly augmented contractility on phenylephrine stimulation, compared with the VSMCs cultured on wells coated with normal human IgG, which was employed as a control (Figure 3a). The augmentation could be neutralized by soluble EPHB4-Fc, which is a preferred receptor of EFNB2, suggesting that among different EPHB
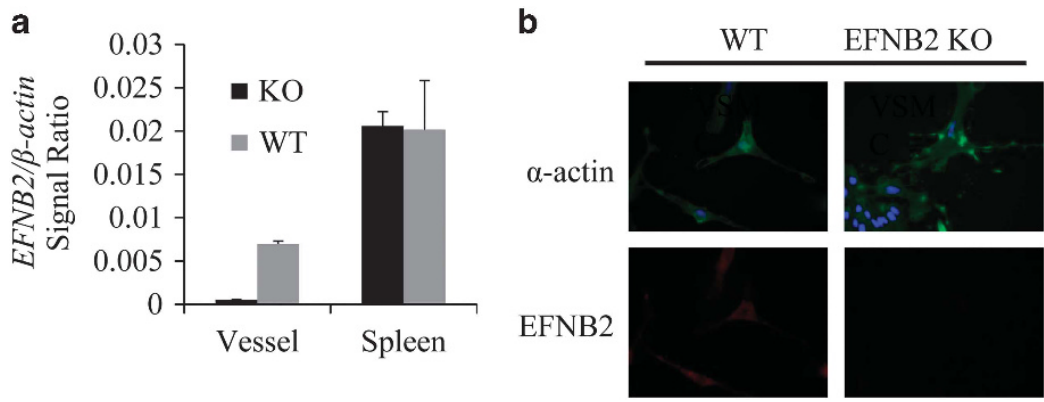

C

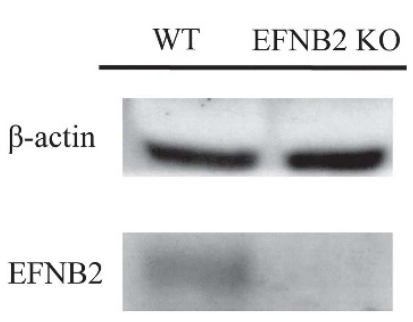

Figure 1 Generation of mice with SMC-specific Efnb2 null mutation. (a) Efnb2 mRNA deletion in mesenteric arteries of EFNB2 KO mice RNA was extracted from the mesenteric arteries and spleens from WT and EFNB2 KO mice and analyzed by RT-qPCR for Efnb2 mRNA levels. $\beta$-actin mRNA levels were used as an internal control. Samples were in triplicate, and means \pm SEM of Efnb2 signal/ $\beta$-actin signal ratios are shown. (b) EFNB2 protein deletion in EFNB2 KO VSMCs according to immunofluorescence microscopy VSMCs from EFNB2 KO and WT mice were isolated from mesenteric arteries, and their EFNB2 (red, lower row) and $\alpha$-actin (green, upper row) expression was detected by immunofluorescence microscopy. (c) EFNB2 protein deletion in EFNB2 KO VSMCs according to immunoblotting VSMCs from EFNB2 KO and WT mice were cultured for 4 days and then collected. Cell lysates were analyzed for EFNB2 protein expression by immunoblotting. The experiments in this figure were conducted three times; representative experiments are shown. 
a
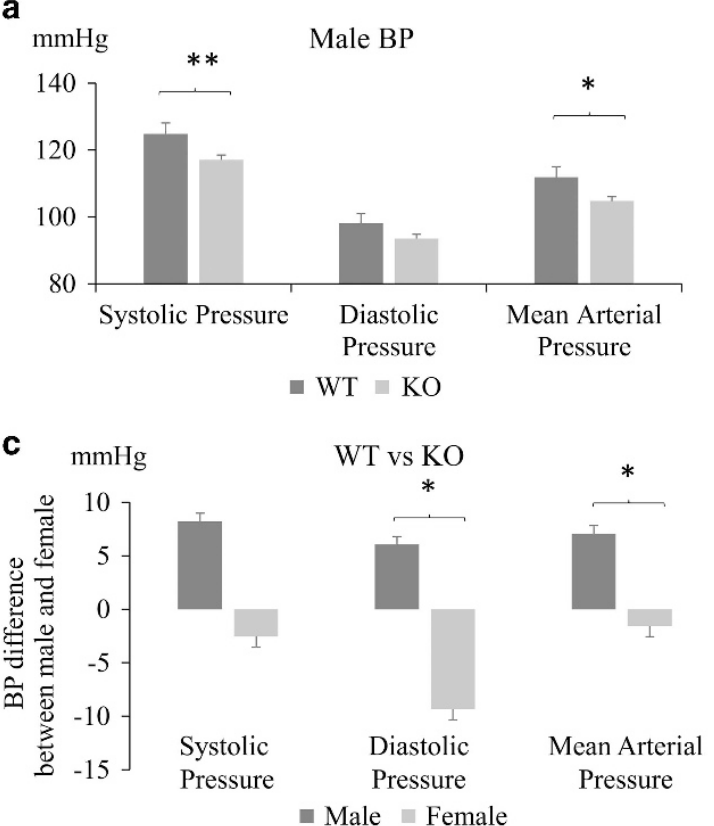

b

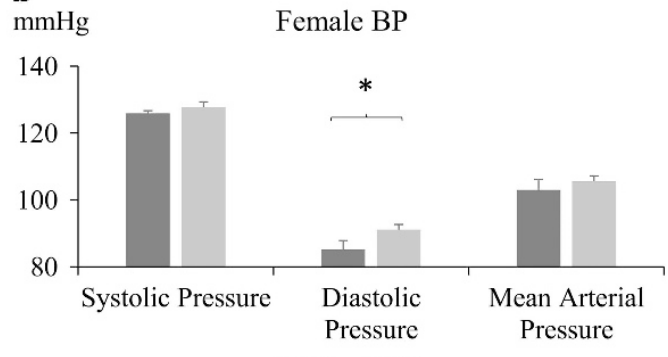

d
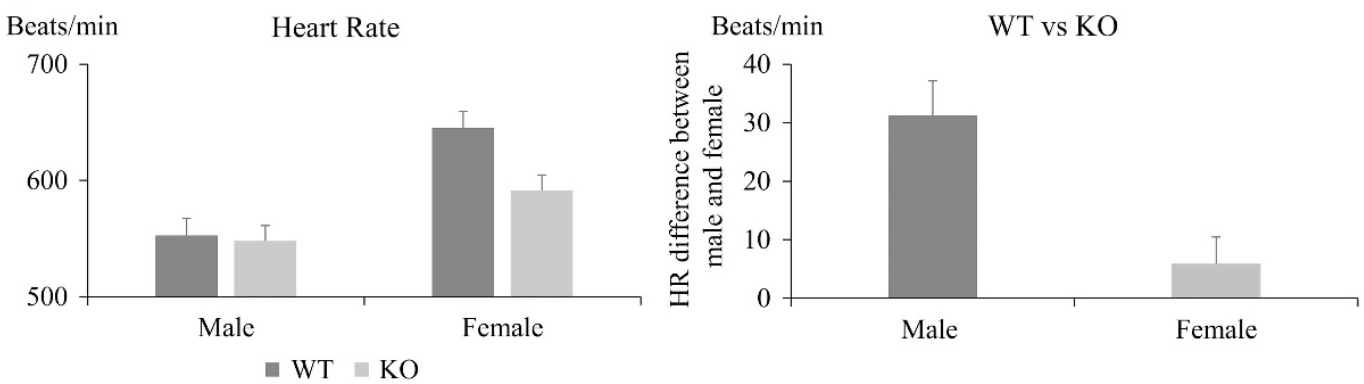

Male: KO 14 weeks $n=5$ WT 14 weeks $n=9$

Female: KO 14 weeks n=9 WT 16 weeks n=9

Figure 2 BP and HR of EFNB2 KO mice. The BP and HR were measured every 2 min by telemetry for $24 \mathrm{~h}$. The mean \pm SEM of daily BP and HR are shown. Parameters of all the time points in the 24-h period were evaluated by a mixed-effects linear model, with genotype, individual mouse, sex and time used as qualitative factors. $P$-values are indicated: ${ }^{*} P<0.05 ;{ }^{*} P<0.01$. The average age of mice of each group at the time of telemetry measurement and the number of each group ( $n$ ) are indicated. (a) BP of male KO and WT mice. (b) BP and of female KO and WT mice. (c) BP differences between KO and WT mice and between males and females. The differences of SP, DP, and MAP of KO vs WT mice (difference $=$ WT value-KO value) are shown. Negative values mean that the KO values are larger than those of WT. The differences between sexes (according to a mixed-effects linear model), if statistically significant, are indicated. (d) HR differences between KO and WT mice (left panel) and between males and females (right panel).

subfamily members, EPHB4 largely mediates forward signaling to achieve increased VSMC contractility. To further prove the essential role of EPHB4 in forward signaling and VSMC contractility, we cultured VSMCs from SMC-specific EPHB4 KO mice ${ }^{27}$ on EFNB2-Fc coated wells. As shown in Figure 3b, solid-phase EFNB2Fc could no longer enhance contractility of EPHB4-deleted VSMCs, again indicating that solid-phase EFNB2's effect on VSMC contractility is largely via EPHB4. To assess whether reverse signaling from EPHBs to EFNB2 also had a role in VSMC contractility, we cultured WT VSMCs on wells coated with anti-EFNB2 Ab to crosslink EFNB2, mimicking the EPHB binding. Such crosslinking could significantly increase VSMC contractility on phenylephrine stimulation (Figure 3c). Moreover, the augmentation could be neutralized by soluble EFNB2-Fc, confirming the specificity of antiEFNB2 Ab. Thus, we have proven that both forward signaling from EFNB2 to EPHB4 and reverse signaling from EPHBs to EFNB2 could enhance VSMC contractility.
To identify the EFNB2 intracellular sequence critical for controlling the VSMC contractility during reverse signaling

EFNB2 has a short intracellular tail (73 aa), which has no enzymatic activity. EFNB2 reverse signaling depends on the association of its intracellular tail with adaptor proteins, which in turn link to various signaling pathways. We conducted step-wise deletion of the EFNB2 intracellular tail from the C-terminus (illustrated in Figure $4 \mathrm{a}$ ) and used lentiviruses to overexpress these deletion mutants in VSMCs from mice with SMC-specific EFNB2 deletion. The infected VSMCs were then cultured in wells coated with anti-EFNB2 Ab. In this system, the absence of endogenous EFNB2 in VSMCs reduced constitutive reverse signaling from EPHB to EFNB2 occurring among neighboring cells, hence reducing the background noise. The overexpression of the exogenous full-length EFNB2 and its deletion mutants were confirmed by RT-qPCR (Figure $4 \mathrm{~b}$ ). The titers of the lentivirus were adjusted such that the deletion mutant mRNA expression was at least the same or $<15 \%$ 


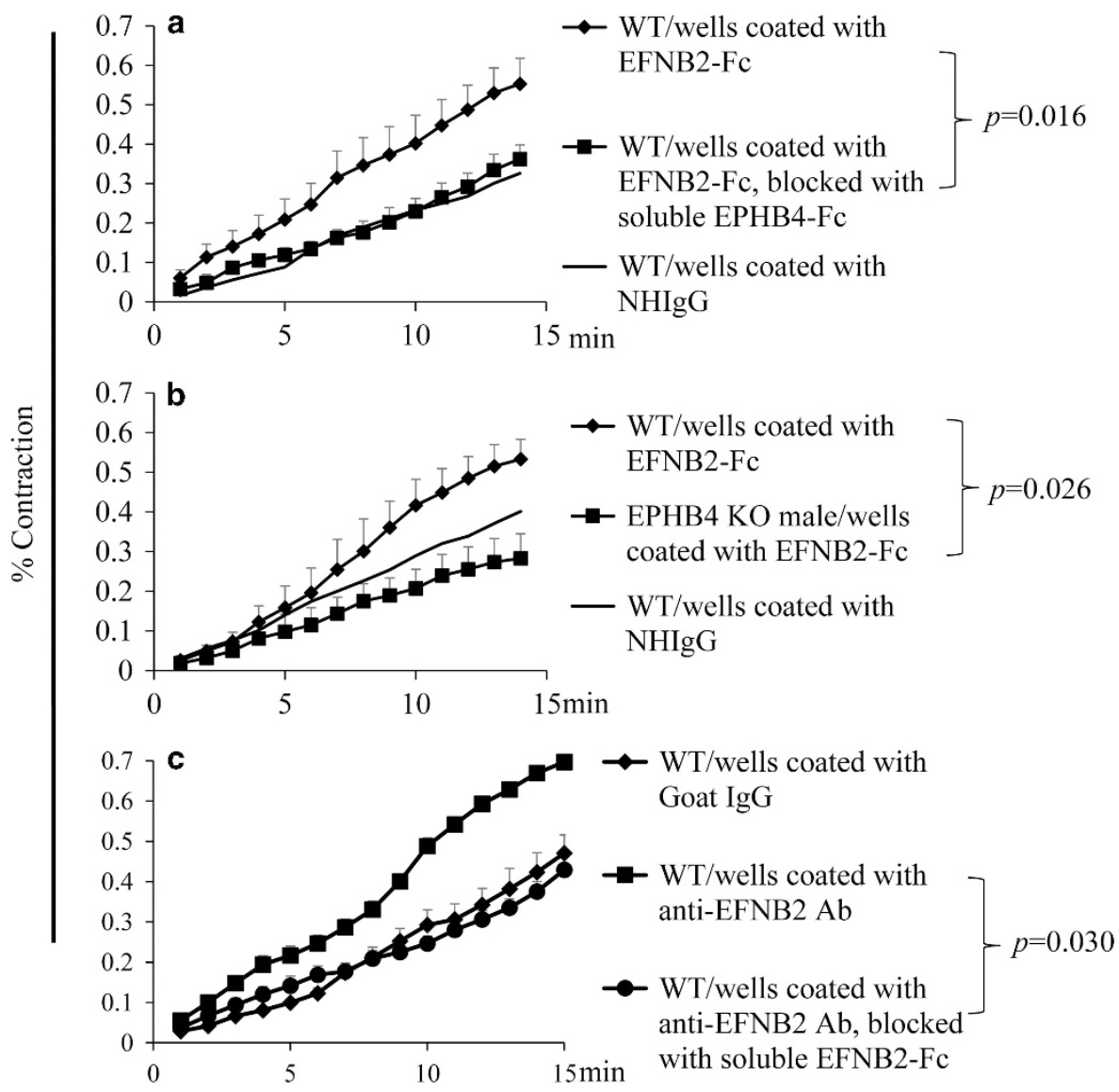

Figure 3 Both forward and reverse signaling by EFNB2 in VSMCs results in increased contractility. Male VSMCs were cultured in wells coated with EFNB2-Fc, normal human IgG (NHIgG; as a control for EFNB2-Fc), goat anti-EFNB2 Ab or goat lgG (as a control for anti-EFNB2 Ab; $2 \mu \mathrm{g} / \mathrm{ml}$ for coating for all the cases) as indicated. VSMCs were stimulated with $20 \mu \mathrm{m}$ phenylephrine at $37^{\circ} \mathrm{C}$ and imaged every min for $15 \mathrm{~min}$. Means \pm SEM of percentage contraction of $15-30$ cells are shown. Data were assessed by one way ANOVA. P-values between groups with significant differences are indicated. All experiments were conducted three times independently. Data from representative experiments are presented. (a) Forward signaling triggered by EFNB2-Fc increases WT VSMC contractility. Male WT VSMCs were cultured in wells coated with EFNB2-Fc or normal human IgG, the latter being presented as a thin line without SEM to facilitate viewing. In one group, soluble EPHB4-Fc $(2 \mu \mathrm{g} / \mathrm{ml})$ was added to the wells to block the interaction between solid-phase EFNB2 and cell surface EPHB4. (b) EPHB4 is mainly responsible for EFNB2-triggered forward signaling for increased VSMC contractility. Male WT or EPHB4 KO VSMCs were cultured in wells coated with EFNB2-Fc, and their contractility on phenylephrine stimulation was measured. Male WT VSMCs cultured in normal human IgG-coated wells were used as an additional control; their mean contractility is presented as a thin line without SEM to facilitate viewing. (c) Reverse signaling mediated by EFNB2 increases WT VSMC contractility. Male WT VSMCs were cultured in wells coated with goat anti-EFNB2 Ab or goat IgG. In one of the groups, soluble EFNB2-FC $(2 \mu \mathrm{g} / \mathrm{ml})$ was added to the culture to block the interaction between solid-phase anti-EFNB2 Ab and cell surface EFNB2 for verification of the Ab specificity.

higher than the full-length Efnb2 mRNA. Overexpression of fulllength EFNB2 (336 a long with 73 aa in the intracellular tail) in EFNB2 KO VSMCs significantly augmented their contractility on PE-stimulation (Figure 4c). The deletion of the last 5 aa in EFNB2 intracellular C-terminus (EFNB2- $\Delta 2 \mathrm{Y}$ ), which removed the PDZ domain-binding motif plus the last two of the total five conserved tyrosine residues (Y333 and Y334), did not reduce VSMC contractility compared with the full-length EFNB2 (Figure 4d). However, an additional deletion of 19-aa, which contains two additional tyrosine residues at Y314 and Y319 (EFNB2- $\Delta 4 \mathrm{Y}$ ), caused a drastic drop of VSMC contractility, which was reduced to the level of control virus-infected VSMCs (Figure 4e). Further deletion of 7 aa, which contains the 5th conserved tyrosine residue Y307 (EFNB2- $\Delta 5 \mathrm{Y}$ ), did not cause more changes compared with EFNB2- $\Delta 4 \mathrm{Y}$ (Figure 4f). These data suggest that the EFNB2 intracellular sequence between aa 313 and aa 331 containing Y314 and Y319 harbors a critical element(s) that could associate with other signaling proteins and regulate VSMC contractility.
Contractility-related signaling events in EFNB2 KO VSMCs We assessed several key signaling events in EFNB2 KO VSMC to further understand the cause of these cells' reduced contractility. VSMCs from male and female KO mice showed mRNA levels of a1adrenoreceptor expression comparable to those from WT counterparts (Supplementary Figure 6A). VSMCs from male KO mice had comparable phenylephrine-stimulated $\mathrm{Ca}^{2+}$ influx to VSMCs from WT mice (Supplementary Figure 6B). Crosslinking EFNB2 with solidphase anti-EFNB2 Ab, which enhanced WT VSMC contractility, did not change phenylephrine-triggered $\mathrm{Ca}^{2+}$ flux in VSMCs from male mice (Supplementary Figure 6C). This indicates that EFNB2 does not affect $\mathrm{Ca}^{2+}$ flux in VSMCs.

The reduced contractility could then be caused by the sensitivity of KO VSMCs to $\mathrm{Ca}^{2+}$ flux. We investigated this possibility by examining KO VSMC myosin light-chain (MLC) phosphorylation, which is known to increase the $\mathrm{Ca}^{2+}$ sensitivity. Indeed, when stimulated with phenylephrine, VSMCs from male but not female KO mice manifested significantly lower MLC phosphorylation at ser19 (Supplementary 
a

Receptor-binding domain

Cytoplasmic

EFNB2 full-length

EFNB2- $\Delta 2 Y$

EFNB2- $\Delta 4 \mathrm{Y}$

EFNB2- $\Delta 5 \mathrm{Y}$

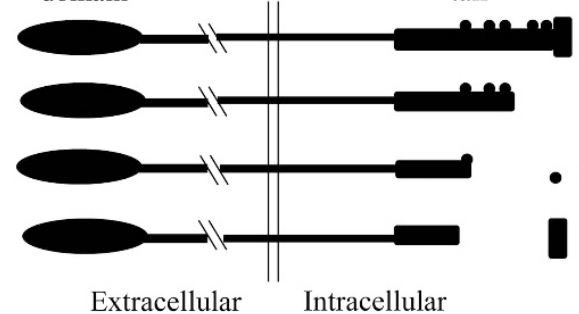

- Tyrosine

PDZ-binding motif

Extracellula

b
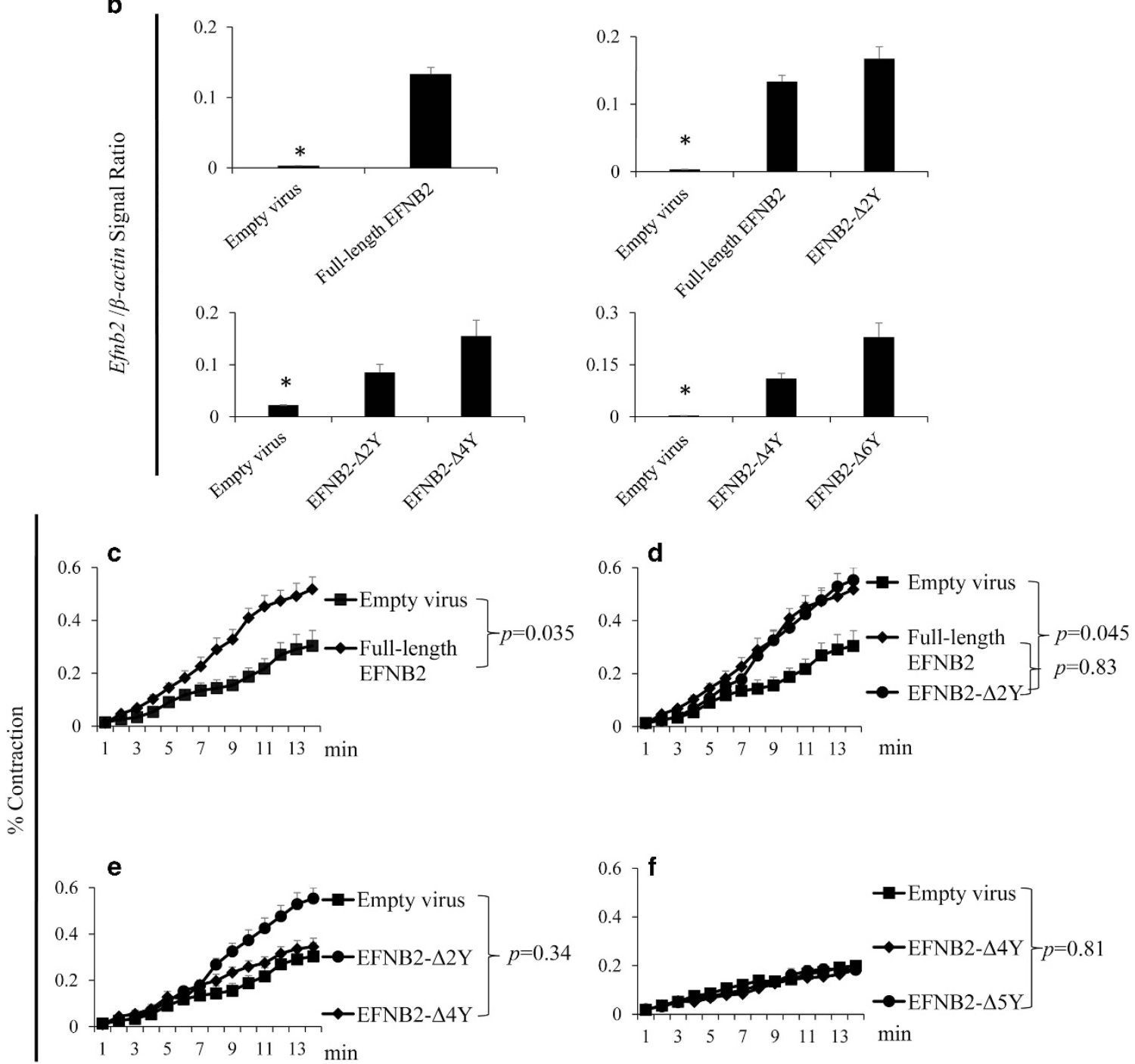

Figure 4 Critical regions in EFNB2 intracellular tail for regulating VSMC contractility. (a) Illustration of EFNB2 deletion mutants the general structures (extracellular domain, intracellular tails, intracellular tyrosine residues (dots) and PDZ domain-binding motif (rectangles)) of EFNB2 are depicted. Different deletion mutants and their nomenclatures are illustrated. (b) mRNA levels of EFNB2 in VSMCs infected with lentiviruses expressing different EFNB2 deletion mutants. Male WT VSMCs were infected with lentiviruses expressing different EFNB2 deletion mutants or the full-length EFNB2, and the mRNA overexpression of these molecules was measured by RT-qPCR, which detected an undeleted region in the EFNB2 sequence. Male WT VSMCs infected with empty viruses were used as a control. Data were expressed as the mean+SEM of ratios of Efnb2 versus $\beta$-actin mRNA signals. The experiment was conducted three or more times, and representative results are shown. ${ }^{*} P<0.05$ (Student's $t$ test). The cells tested in $B$ were the same ones used in $\mathbf{c}-\mathbf{f}$. (c-f) Identification of a critical region in the EFNB2 intracellular tail for regulating VSMC contractility. Male EFNB2 KO VSMCs were infected with lentiviruses expressing the full-length EFNB2 or its deletion mutants, and the VSMCs were cultured in wells coated with anti-EFNB2 Ab. VSMC contractility on phenylephrine stimulation $(20 \mu \mathrm{m})$ was measured. Means+SEM of percentage contraction of 15-30 cells are shown. Data were assessed by ANOVA. $P$-values between groups with significant differences are indicated. Data from representative experiments are presented. 
Figure 6D), consistent with reduced $\mathrm{BP}$ in male but not female $\mathrm{KO}$ mice (Figures 2a and b). Myosin light chain is dephosphorylated by myosin phosphatase. Phosphorylation of myosin phosphatase target protein-1 (thr696), a process that decreases myosin light chain phosphatase activity, ${ }^{30}$ was not influenced by EFNB2 deletion (Supplementary Figure 6E). ERK activation can enhance smooth muscle activation through several proposed modes of action: (1) it might activate MLC kinase through a so-far un-delineated pathway $^{31,32}$ or (2) it might phosphorylate the actin-binding protein, caldesmon, which removes caldesmon's inhibitory effect on smooth muscle contraction. ${ }^{33}$ In both male and female, KO VSMCs, ERK phosphorylation, a surrogate marker of ERK activation, was significantly reduced on phenylephrine stimulation (Supplementary Figure 6F), implicating ERK as a link in EFNB2 deletion-caused hypocontractility of VSMCs. The lack of sex difference in ERK phosphorylation suggests that although it might contribute to reduce VSMC contractility in KO mice, it is not responsible for the observed sex difference.

Association of EFNB2 with hypertension in subjects with diabetes We tested the association of 55 DNA markers falling within the EFNB2 gene or within additional $\sim 10 \mathrm{~kb} 5^{\prime}$ and $10 \mathrm{~kb} 3^{\prime}$ of the gene (located within a 58.72-kb region between positions 107134861 and 107193584 on chromosome 13 (Build37/hg19) with hypertension in a type 2 diabetes patient cohort; males and females were tested separately. Five SNPs were found to be significantly associated with hypertension for the male-only samples (Table 1), using a Bonferronicorrected critical $P$-value of $3.33 \times 10^{-3}$ for 15 independent tests, corresponding to 15 independent $\left(r^{2} \leq 0.80\right)$ LD blocks within the region. The locations of the five significant SNPs (encircled) with respect to the LD structure around the $3^{\prime}$ end of the EFNB2 gene are shown in Supplementary Figure 7A. These SNPs fall just downstream (within $1 \mathrm{~kb}$ ) of the $3^{\prime}$ untranslated region (UTR) of the gene. The five SNPs, with the most significant SNP, rs7328882, as a representative, whose allele frequencies are depicted in Supplementary Figure $7 \mathrm{~B}$, are all in high $\mathrm{LD}\left(r^{2} \geq 0.80\right)$, and so are likely to all be proxy to one underlying functional polymorphism located within the $3^{\prime}$ region of the gene or just $3^{\prime}$ downstream of the gene.

The coding (minor) allele of these five significantly associated SNPs in male subjects was protective, as their odds ratios were below 1, and the $95 \%$ CIs of the odds ratios did not include 1 (Table 1). When chronic kidney disease (CKD), defined as estimated glomerular filtration rate $<60 \mathrm{ml} / \mathrm{min} / 1.73 \mathrm{~m}^{2}$, was used as a covariate in the logistic association model, the $P$-values were slightly increased, although the general pattern of association remained the same; however, only rs7328882, which was the most significant SNP for the model without $\mathrm{CKD}$ as a covariate, remained significant at the Bonferroni-corrected critical $P$-value (Table 1). No SNPs were nominally significant for the female-only samples in either the case of inclusion or exclusion of CKD as a covariate (Table 1). The CIs of odds ratios of SNPs for the females-only samples all included 1 . These confidence intervals were wider $(\sim 0.45)$ for females than for the male samples $(\sim 0.25)$, likely reflecting lower statistical power due to a smaller female sample size. The differences in odds ratios between male and female subjects calculated using the Altman-Bland test ${ }^{34}$ for SNP, rs7328882, was not significant $\left(P=7.98 \times 10^{-2}\left(<1 \times 10^{-2}\right.\right.$ but $>5 \times 10^{-2}$ ), most likely due to lack of power in the female samples.

\section{DISCUSSION}

$\mathrm{BP}$ is a vital physiological parameter and is highly regulated with multiple compensatory mechanisms including blood volume, cardiac output and/or vascular tone regulation that maintain it within a normal range. EFNB2 deletion in VSMCs likely leads to a default phenotype of reduced VSMC contractility, and consequently reduced

Table 1 SNP rs numbers, alleles, allele frequencies, $P$-values, odds ratios and $95 \%$ confidence intervals (Cls) of odds ratios for SNPs associated with hypertension in the ADVANCE study with normal $P$-values $\leqslant 5.00 \times 10^{-2}$ for male-only or female-only samples with CKD included and not included in the covariates for the analysis

\begin{tabular}{|c|c|c|c|c|c|c|c|c|c|c|c|c|}
\hline \multirow[b]{3}{*}{ SNP } & \multirow[b]{3}{*}{$\begin{array}{c}\text { Position chrome } 13 \\
\text { (Build37/ hg19) }\end{array}$} & \multirow[b]{3}{*}{$\begin{array}{l}\text { Coding } \\
\text { allele }\end{array}$} & \multirow[b]{3}{*}{$\begin{array}{c}\text { Alternate } \\
\text { allele }\end{array}$} & \multirow[b]{3}{*}{$\begin{array}{l}\text { Frequency of } \\
\text { coding allele }\end{array}$} & \multicolumn{4}{|c|}{ Males } & \multicolumn{4}{|c|}{ Females } \\
\hline & & & & & & & Lower & & & & Lower & \\
\hline & & & & & $\begin{array}{l}\text { Association } \\
\text { P-value }^{\mathrm{b}}\end{array}$ & $\begin{array}{l}\text { Odds } \\
\text { ratio }\end{array}$ & $\begin{array}{l}95 \% \\
\mathrm{Cr}\end{array}$ & $\begin{array}{l}\text { Upper } \\
95 \% \mathrm{CF}\end{array}$ & $\begin{array}{l}\text { Association } \\
\text { P-value }\end{array}$ & $\begin{array}{l}\text { Odds } \\
\text { ratio }\end{array}$ & $\begin{array}{l}95 \% \\
\mathrm{Cr}\end{array}$ & $\begin{array}{l}\text { Upper } \\
95 \% \mathrm{Cr}\end{array}$ \\
\hline \multicolumn{13}{|c|}{ Without CKD as a covariate } \\
\hline rs9555251 & 107140587 & $\mathrm{~T}$ & C & 0.35 & $2.23 \times 10^{-3}$ & 0.78 & 0.66 & 0.91 & $8.77 \times 10^{-1}$ & 0.98 & 0.78 & 1.24 \\
\hline rs7322914 & 107140910 & $\mathrm{~T}$ & C & 0.35 & $2.19 \times 10^{-3}$ & 0.78 & 0.66 & 0.91 & $8.69 \times 10^{-1}$ & 0.98 & 0.78 & 1.23 \\
\hline rs7328698 & 107141349 & C & G & 0.35 & $1.88 \times 10^{-3}$ & 0.77 & 0.66 & 0.91 & $8.02 \times 10^{-1}$ & 0.97 & 0.77 & 1.22 \\
\hline rs7328882 & 107141433 & $A$ & G & 0.35 & $1.13 \times 10^{-3}$ & 0.76 & 0.65 & 0.90 & $8.78 \times 10^{-1}$ & 0.98 & 0.78 & 1.23 \\
\hline rs7329357 & 107141705 & $A$ & G & 0.35 & $1.73 \times 10^{-3}$ & 0.77 & 0.65 & 0.91 & $8.62 \times 10^{-1}$ & 0.98 & 0.78 & 1.23 \\
\hline rs9520087 & 107144320 & $A$ & G & 0.43 & $9.35 \times 10^{-3}$ & 0.81 & 0.69 & 0.95 & $7.69 \times 10^{-1}$ & 1.03 & 0.83 & 1.29 \\
\hline \multicolumn{13}{|c|}{ With CKD as a covariate } \\
\hline rs9555251 & 107140587 & $\mathrm{~T}$ & C & 0.35 & $4.62 \times 10^{-3}$ & 0.79 & 0.67 & 0.93 & $6.29 \times 10^{-1}$ & 0.94 & 0.74 & 1.20 \\
\hline rs7322914 & 107140910 & $\mathrm{~T}$ & $\mathrm{C}$ & 0.35 & $4.57 \times 10^{-3}$ & 0.79 & 0.67 & 0.93 & $6.21 \times 10^{-1}$ & 0.94 & 0.74 & 1.19 \\
\hline rs7328698 & 107141349 & C & G & 0.35 & $4.12 \times 10^{-3}$ & 0.79 & 0.67 & 0.93 & $5.60 \times 10^{-1}$ & 0.93 & 0.74 & 1.18 \\
\hline rs7328882 & 107141433 & $A$ & G & 0.35 & $2.54 \times 10^{-3}$ & 0.78 & 0.66 & 0.91 & $6.27 \times 10^{-1}$ & 0.94 & 0.75 & 1.19 \\
\hline rs7329357 & 107141705 & $A$ & G & 0.35 & $3.91 \times 10^{-3}$ & 0.78 & 0.66 & 0.92 & $6.99 \times 10^{-1}$ & 0.95 & 0.76 & 1.21 \\
\hline rs9520087 & 107144320 & $A$ & G & 0.43 & $1.78 \times 10^{-2}$ & 0.82 & 0.70 & 0.97 & $9.03 \times 10^{-1}$ & 1.01 & 0.81 & 1.28 \\
\hline
\end{tabular}

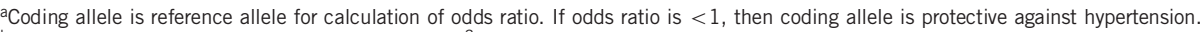

b $P$-values $\leq$ Bonferroni critical $P$-value of $3.33 \times 10^{-3}$ are indicated in bold.

c95\% Cl: $95 \%$ confidence interval for odds ratio. 
BP. This phenotype is pronounced in males, with SP and MAP reaching statistical significance. However, in female $\mathrm{KO}$ mice, $\mathrm{BP}$ parameters (SP, DP and MAP) were not reduced, with DP being significantly increased. This suggests that one or more of the abovementioned compensation mechanisms is at work, and sex/sex hormone might be an additional modifier, which could even push the final outcome to a different direction. The nature of the sex/sex hormone-dependent modulation of BP in the absence of EFNB2 remains to be investigated. We have previously described that a significant portion of cardiometabolic phenotypes has a sex-dependent penetrance in families with hypertension on Quebec founder population. ${ }^{35}$ Regardless, the sex difference in the BP phenotype of the $\mathrm{KO}$ mice is true and significant, according to analysis with a mixed-effects linear model (Figure 2c).

Nakayama et al. ${ }^{36}$ reported that smooth muscle-specific deletion of EFNB2 using SM22 $\Delta$-Cre results in lower body weight, reduced VSMC proliferation, thinner arterial vessel wall and enlarged arterial diameter. However, none of these phenotypes was observed in our $\mathrm{KO}$ mice in which EFNB2 was deleted using smMHC-Cre. There could be several possible explanations for these discrepancies. (1) The mouse genetic background might heavily influence these phenotypes: Nakayama's mice are in a $129 \mathrm{sv} \times \mathrm{C} 57 \mathrm{BL} / 6 \mathrm{~J}$ mixed background, whereas ours are in a pure C57BL/6J background. (2) Different smooth musclespecific promoters might result in different degrees of EFNB2 deletion in target organs/tissues and of leaky deletion in untargeted organs/ tissues. (3) Cell proliferation was assessed at different time points (postnatal day 8 in Nakayama's study $v$ s adult in our study). Although Nakayama's study focuses on EFNB2's role in PGDFR $\beta$ signaling in VSMCs related to their proliferation, we are focusing on VSMCs' functionality and their signaling during contractile stimulation. Naturally, these two aspects have different time windows. Nevertheless, these two studies agree that EFNB2 has a critical role in VSMC biology, which has not been previously appreciated.

EFNB2 can transduce signals in both forward and reverse directions. We have demonstrated that both directions are involved in VSMC contractility. EPHB4 is the preferred EPH, with which EFNB2 could associate. We have proved in vitro that only WT, but not EPHB4 KO VSMCs, could respond to EFNB2 stimulation and augment contractility (Figure $3 \mathrm{~b}$ ), indicating that the predominant effect of EFNB2 forward signaling with regard to enhancing VSMC contractility was mediated by EPHB4. If this is the case, then SMC-specific deletion of EPHB4 should have a similar BP phenotype to that seen in the EFNB2 KO mice. Indeed, our recent report reveals that EPHB4 $\mathrm{KO}$ mice present sex-dependent hypotension, with male KO being hypotensive, whereas female KO being normotensive, ${ }^{27}$ corroborating our results from EFNB2 $\mathrm{KO}$ mice.

EFNB2 has a short intracellular tail (73 aa), which has no enzymatic activity. Its reverse signaling depends on adaptor proteins associating with the tail. EFNB2 intracellular tails are characterized by two major features: the C-terminal PDZ domain-binding motif and five conserved tyrosine residues, which could be associated with PDZ domaincontaining proteins (eg, PDZ-RGS3, GRIP1/2, TIAM1 and DISHEVELED) $)^{3,17,21,37,38}$ and $\mathrm{SH} 2$ domain-containing proteins (eg GRB4, STAT3 and CRK, ${ }^{39}$ respectively). These two features are located in the last 31 aa, which are highly conserved. EFNB can also have PDZ- and $\mathrm{SH} 2$-independent functions, ${ }^{40}$ some of which presumably depend on the SH3 domain of associating proteins (eg, CRK and GRB4). By deleting different lengths of the EFNB2 intracellular tail, we found that the C-terminal PDZ domain-binding motif and the last two tyrosine residues were dispensable for EFNB2 reverse signaling with regard to VSMC contractility, but a 19-aa long region (ie, from aa 313 to aa 331) containing the $3 \mathrm{rd}$ and 4 th conserved tyrosine residues (counting from the C-terminus) was critical. The two tyrosine residues (Y314 and Y319) in this region are good candidates responsible for reverse signaling as they could interact with $\mathrm{SH} 2$ domain-containing adaptor proteins mentioned above, although S328 should also be considered, as it may attract $\mathrm{SH} 3$ domain-containing adaptor proteins. It is possible that the molecules associating with these residues are connected to the VSMC contraction machinery, and the function of these associating molecules is differentially affected by sex hormones in the absence or presence of EFNB2. A search of the associated proteins that mediate EFNB2's effect and a study of how their effects are influenced by sex hormones with regard to VSMC contractility are warranted.

Our human genetic study revealed that five SNPs that are in high LD with each other in the $3^{\prime}$ untranslated region of EFNB2 were significantly associated with hypertension risk at a $P$-value below the Bonferroni-corrected $P$-value in the males-only samples but not in females-only samples. The minor alleles of each of the five significant SNPs were negatively associated with hypertension risk (ie, protective against hypertension, with odds ratios between 0.77 and 0.78 ) in male human subjects. These SNPs are likely in LD with a functional mutation that may be linked to the effects of EFNB2 on BP through the $3^{\prime}$ UTR; however, additional functional studies are required to investigate this possibility. This finding implies that the occurrence of a loss-of-function mutation in the EFNB2 gene, in LD with the coding allele of the five associated SNPs, could result in reduced risk of hypertension in male patients, and this is not inconsistent with the effect of EFNB2 deletion in mice. However, it must be emphasized that a human gene mutation is not equivalent to the mouse EFNB2 deletion, which invariably leads to loss-of-function; a human mutation could be either a loss- or gain-of-EFNB2-function. A gain-of-function of mutation in human males could explain the association of increased hypertension risks with EFNB2 mutations in the alternative allele.

Our mouse study showed that in females, EFNB2 null mutation did not lead to hypotension. Therefore, EFNB2 gene mutation in female humans should not impact on BP, or at least should not affect the BP in the same direction as it would in the males, if our mouse study is of any guidance. Indeed, we observed no association between EFNB2 SNPs with hypertension in females in this study. The mechanisms responsible for the influence of sex/sex hormones in regulating BP jointly with EFNB2 are currently under investigation. With that said, our results of the Altman-Bland test show that there is no difference between males-only and females-only samples with regards to the odds ratios of the five SNPs (the $P$-value of the most significant SNPs rs7328882 was given in the 'Results' section; $P$-values of other SNPs are not shown). A possible explanation is that the female sample size is rather small; with a larger sample size, we might observe a significant difference after the Altman-Bland test, as would be expected.

As male but not female EFNB2 KO mice had lower BP, logically this suggests that either testosterone is inductive to the lower BP when EFNB2 is absent, or else EFNB2 deletion has a default effect lowering BP but estrogen counteracts this effect. With this conceptual frame work, we attempt to explain the detection of association of EFNB2 SNPs with hypertension in males in the ADVANCE genetic study in which all the cases and controls are type 2 diabetics.

The minimal age of male patients in the ADVANCE study was 55 years. Plasma total testosterone levels range from 270 to $1070 \mathrm{ng} / \mathrm{dl}$ in 'normal' males. Considering $346 \mathrm{ng} / \mathrm{dl}$ as a cut-off for the diagnosis of hypogonadism, as recommended by the International Society for the Study of the Aging Male, about 30\% of men older than 40 years are hypogonadic. ${ }^{41}$ Further, male patients with the metabolic 
syndrome are prone to hypogonadism. ${ }^{42}$ Therefore, it is conceivable that more than $30 \%$ of the male patients in the ADVANCE study suffer from hypogonadism.

Further, the type 2 diabetes males are generally overweight or obese. Adipose tissues are rich in aromatase, a rate-limiting enzyme converting testosterone to estrogen. ${ }^{43}$ Hence, overweight/obese males tend to have relatively higher estrogen levels locally in the perivascular adipose tissues or systemically.

The above-described special changes of sex hormone levels in the ADVANCE males might make them a unique subpopulation in which the association between EFNB2 mutations and hypertension risks becomes easier to detect, compared with the general population. Therefore, the observed sex difference in EFNB2 SNPs with regard to their association to hypertension might exist in certain subpopulations based on sample selection, and further validation studies of this explanation is warranted.

The incidence of CDK (including diabetic nephropathy) is increased in diabetic patients. ${ }^{44}$ As CDK is a cause of hypertension, it is therefore logical to include CDK as a covariate, to exclude the possibility that EFNB2 is in association with hypertension via CDK. We registered the CDK incidence in our cohorts, and the data are presented in Supplementary Table 4. Generally speaking, there are more subjects without CKD than with CKD, for both males and females (Supplementary Table 4A). In the male cohort, 16 and $9 \%$ of the hypertensive subjects and normotensive subjects, respectively, have CKD (Supplementary Table 4B), showing significant dependence between hypertension and CKD. In the female cohort, however, there is no significant dependence between hypertension and CKD (Supplementary Table 4C). In our genetic study, five EFNB2 SNPs are significantly associated with hypertension in males but not females. Does such association depend on CKD? The answer is negative based on following considerations. (1) After CKD was used as a covariate, one SNP rs7328882 was still significantly associated with hypertension in males, and the rest four SNPs, although their $P$-values increased, but were just above the Bonferroni-corrected $P$-value. Considering the conservative nature of Bonferroni correction, and the high LD link of rs7328882 with the other four SNPs, it is reasonable to assume that the functional mutation related to this LD block is still significantly associated with hypertension even after CKD was used a covariate. (2) Although CKD could cause hypertension, ${ }^{45}$ the reverse is also true, ie, hypertension frequently leads to the development of CKD. ${ }^{46}$ In our case, the introduction of CKD as a covariate in the statistical analysis reduces somewhat significance of the association between some EFNB2 SNPs and hypertension, but this does not necessarily mean that CKD is causative for the hypertension. Rather, as CKD and hypertension phenotypes are correlated and confounded, we might have simply removed some weight of hypertension and reduced the power of the analysis. (3) Our human genetic study is corroborated by the KO mouse phenotype, which is caused by vascular defects but not kidney dysfunction. Taking together, we believe that the association of EFNB2 SNPs to hypertension is direct but not via CKD.

We have queried the International Consortium for Blood Pressure (ICBP) dataset, ${ }^{47}$ but no significant association of EFNB2 SNPs with BP is found. We should point out the differences in the two studies. The IBPC sample specifically excluded, cohorts of diabetic cases, hypertensive cases, and myocardial infarction cases, which is specifically the type of samples we analyzed. They analyzed general population samples that had incidentally been measured for SP and $\mathrm{DP}$, and performed a quantitative analysis against DP and SP values. The advantage of their approach is that it allows the assembly of very large sample sizes to increase power; however, it also might introduce significant heterogeneity into their sample decreasing genotype relative risks. In our current study, we used a qualitative phenotype that defined cases and controls for hypertension. Also, multiple BP measurements were carefully made over multiple time points in the course of the ADVANCE study, and only consistently high readings were included as cases.

The five associated SNPs are in LD with each other and are located within a large LD block that includes the 3' UTR region of EFNB2 and could reflect the presence a polymorphism within the $3^{\prime}$ untranslated region (3'-UTR) of the gene. $3^{\prime}$ UTRs of various genes are known to contain regulatory elements that can control rates of translation and mRNA stability by serving as binding sites for various proteins or miRNAs. Many 3'-UTRs contain AU-rich elements which affect the stability or decay rate of transcripts in a localized manner or affect translation initiation. Also the 3'-UTR contains the sequence AAUAAA that directs addition of the poly-A tail, which is involved in mRNA stability. By binding to specific sites within the $3^{\prime}$-UTR, miRNAs can decrease gene expression of various mRNAs by either inhibiting translation or directly causing degradation of the transcript. Discovery of the exact location and mechanism of the functional mutations in LD with the identified SNPs would require further extensive functional research.

This study and our most recent publication show that the deletion of either of the receptor EPHB4 or its major ligand EFNB2 leads to a similar hypotensive phenotype in mice, ${ }^{27}$ corroborating the results of our human genetics study. Collectively, these novel findings reveal a previously unknown EPH/EFN-based mechanism for BP regulation.

\section{CONFLICT OF INTEREST}

The authors declare no conflict of interest.

\section{ACKNOWLEDGEMENTS}

This work was supported by grants from the Canadian Institutes of Health Research to JW (MOP57697, MOP69089 and MOP 123389), HL (MOP97829), ET (MOP14496) and JT (ISO106797). It was also funded by grants from the Heart and Stroke Foundation of Quebec, the Natural Sciences and Engineering Research Council of Canada (203906-2012), the Juvenile Diabetes Research Foundation (17-2013-440), the Fonds de recherche du Québec-Santé (Ag-06) and the J-Louis Lévesque Foundation to JW.

\footnotetext{
1 Unified nomenclature for Eph family receptors and their ligands, the ephrins. Eph Nomenclature Committee. Cell 1997; 90: 403-404.
}

2 Pasquale EB: Eph-ephrin bidirectional signaling in physiology and disease. Cell 2008; 133: 38-52.

3 Luo H, Yu G, Tremblay J, Wu J: EphB6-null mutation results in compromised T cell function. J Clin Investig 2004; 114: 1762-1773.

4 Batlle E, Henderson JT, Beghtel $\mathrm{H}$ et al: Beta-catenin and TCF mediate cell positioning in the intestinal epithelium by controlling the expression of EphB/ephrinB. Cell 2002; 111: 251-263.

5 Konstantinova I, Nikolova G, Ohara-Imaizumi M et al: EphA-Ephrin-A-mediated beta cell communication regulates insulin secretion from pancreatic islets. Cell 2007; 129: 359-370.

6 Salvucci O, de la Luz Sierra M, Martina JA et al: EphB2 and EphB4 receptors forward signaling promotes SDF-1-induced endothelial cell chemotaxis and branching remodeling. Blood 2006; 108: 2914-2922.

7 Luo $\mathrm{H}$, Broux B, Wang $\mathrm{X}$ et al: EphrinB1 and EphrinB2 regulate $\mathrm{T}$ cell chemotaxis and migration in experimental autoimmune encephalomyelitis and multiple sclerosis. Neurobiol Dis 2016; 91: 292-306.

8 Kaushal GP, Shah SV: The new kids on the block: ADAMTSs, potentially multifunctional metalloproteinases of the ADAM family. J Clin Investig 2000; 105: 1335-1337.

9 Primakoff P, Myles DG: The ADAM gene family: surface proteins with adhesion and protease activity. Trends Genet 2000; 16: 83-87.

10 Georgakopoulos A, Litterst C, Ghersi E et al: Metalloproteinase/Presenilin1 processing of ephrinB regulates EphB-induced Src phosphorylation and signaling. Embo J 2006; 25: $1242-1252$. 
11 Tomita T, Tanaka S, Morohashi Y et al: Presenilin-dependent intramembrane cleavage of ephrin-B1. Mol Neurodegener 2006; 1: 2.

12 Xin HB, Deng KY, Rishniw M et al: Smooth muscle expression of Cre recombinase and eGFP in transgenic mice. Physiol Genomics 2002; 10: 211-215.

13 Dumas P, Pausova Z, Kren V et al: Contribution of autosomal loci and the $Y$ chromosome to the stress response in rats. Hypertension 2000; 35: 568-573.

14 Luo $\mathrm{H}$, Charpentier $\mathrm{T}$, Wang $\mathrm{X}$ et al: Efnb1 and Efnb2 proteins regulate thymocyte development, peripheral T cell differentiation, and antiviral immune responses and are essential for interleukin-6 (IL-6) signaling. J Biol Chem 2011; 286: 41135-41152.

15 Luo $\mathrm{H}$, Wan X, Wu Y et al: Cross-linking of EphB6 resulting in signal transduction and apoptosis in Jurkat cells. J Immunol 2001; 167: 1362-1370.

16 Luo H, Wu Z, Qi S et al: Ephrinb1 and Ephrinb2 are associated with interleukin-7 receptor alpha and retard its internalization from the cell surface. J Biol Chem 2011; 286: 44976-44987.

17 Luo $\mathrm{H}, \mathrm{Yu} \mathrm{G}, \mathrm{Wu} \mathrm{Y}$ et al: EphB6 crosslinking results in costimulation of $\mathrm{T}$ cells. J Clin Investig 2002; 110: 1141-1150.

$18 \mathrm{Wu} \mathrm{J}$, Luo H: Recent advances on T-cell regulation by receptor tyrosine kinases. Curr Opin Hematol 2005; 12: 292-297.

19 Yu G, Luo H, Wu Y et al: Ephrin B2 induces T cell costimulation. J Immunol 2003, 171: $106-114$.

$20 \mathrm{Yu} \mathrm{G}$, Luo H, Wu Y et al: Mouse ephrinB3 augments T-cell signaling and responses to T-cell receptor ligation. J Biol Chem 2003; 278: 47209-47216.

21 Yu G, Luo H, Wu Y et al: EphrinB1 is essential in T-cell-T-cell co-operation during T-cell activation. J Biol Chem 2004; 279: 55531-55539.

22 Yu G, Mao J, Wu Y et al: Ephrin-B1 is critical in T-cell development. J Biol Chem 2006; 281: 10222-10229.

23 Hashimoto T, Karasawa T, Saito A et al: Ephrin-B1 localizes at the slit diaphragm of the glomerular podocyte. Kidney Int 2007; 72: 954-964.

24 Dravis $\mathrm{C}, \mathrm{Wu} \mathrm{T}$, Chumley MJ et al: EphB2 and ephrin-B2 regulate the ionic homeostasis of vestibular endolymph. Hear Res 2007; 223: 93-104.

25 Luo H, Wu Z, Tremblay J et al: Receptor tyrosine kinase Ephb6 regulates vascular smooth muscle contractility and modulates blood pressure in concert with sex hormones. J Biol Chem 2012; 287: 6819-6829.

$26 \mathrm{Wu}$ Z, Luo H, Thorin E et al: Possible role of Efnb1 protein, a ligand of Eph receptor tyrosine kinases, in modulating blood pressure. J Biol Chem 2012; 287: 15557-15569.

27 Wang $\mathrm{Y}$, Thorin $\mathrm{E}$, Luo $\mathrm{H}$ et al: EPHB4 protein expression in vascular smooth muscle cells regulates their contractility, and EPHB4 deletion leads to hypotension in mice. J Biol Chem 2015; 290: 14235-14244.

28 Colagiuri S, Cooper M, Grobbee R et al: Prevention of Vascular Outcomes in Type 2 Diabetes: Benefits of Intensive Blood Pressure and Blood Glucose Control with Preterax and Diamicron MR, Issue III. France: Wolters Kluwer Health France, 2008.
29 Advance Trial/Action in Diabetes and Vascular Disease: PreterAx and Diamicron MR Controlled Evaluation. Available at: www.advance-trial.com. Accessed on November 2014.

30 Ramachandran C, Patil RV, Combrink $\mathrm{K}$ et al: Rho-Rho kinase pathway in the actomyosin contraction and cell-matrix adhesion in immortalized human trabecular meshwork cells. Mol Vis 2011; 17: 1877-1890.

31 Roberts RE: The role of Rho kinase and extracellular regulated kinase-mitogenactivated protein kinase in alpha2-adrenoceptor-mediated vasoconstriction in the porcine palmar lateral vein. J Pharmacol Exp Therap 2004; 311: 742-747.

32 Klemke RL, Cai S, Giannini AL et al: Regulation of cell motility by mitogen-activated protein kinase. J Cell Biol 1997; 137: 481-492.

33 Hedges JC, Oxhorn BC, Carty $M$ et al: Phosphorylation of caldesmon by ERK MAP kinases in smooth muscle. Am J Physiol Cell Physiol 2000; 278: C718-C726.

34 Altman DG, Bland JM: Interaction revisited: the difference between two estimates. Bmj 2003; 326: 219.

35 Seda 0, Tremblay J, Gaudet D et al: Systematic, genome-wide, sex-specific linkage of cardiovascular traits in French Canadians. Hypertension 2008; 51: 1156-1162.

36 Nakayama A, Nakayama M, Turner CJ et al: Ephrin-B2 controls PDGFRbeta internalization and signaling. Genes Dev 2013; 27: 2576-2589.

37 Bruckner K, Pasquale EB, Klein R: Tyrosine phosphorylation of transmembrane ligands for Eph receptors. Science 1997; 275: 1640-1643.

38 Cowan CA, Henkemeyer M: The SH2/SH3 adaptor Grb4 transduces B-ephrin reverse signals. Nature 2001; 413: 174-179.

39 Arvanitis D, Davy A: Eph/ephrin signaling: networks. Genes Dev 2008; 22: 416-429.

40 Lu Q, Sun EE, Klein RS et al: Ephrin-B reverse signaling is mediated by a nove PDZ-RGS protein and selectively inhibits G protein-coupled chemoattraction. Cell 2001; 105: 69-79.

41 Traish AM, Miner MM, Morgentaler A et al: Testosterone deficiency. Am J Med 2011 ; 124: 578-587.

42 Zitzmann M: Testosterone deficiency, insulin resistance and the metabolic syndrome. Nat Rev Endocrinol 2009; 5: 673-681.

43 Nelson LR, Bulun SE: Estrogen production and action. J Am Acad Dermatol 2001; 45 S116-S124.

44 National Kidney Foundation: K/DOQI clinical practice guidelines for chronic kidney disease: evaluation, classification, and stratification. Am J Kidney Dis 2002; 39: S1-266.

45 Schiffrin EL, Lipman ML, Mann JF: Chronic kidney disease: effects on the cardiovascular system. Circulation 2007; 116: 85-97.

46 Klag MJ, Whelton PK, Randall BL et al: Blood pressure and end-stage renal disease in men. N Engl J Med 1996; 334: 13-18.

47 International Consortium for Blood Pressure Genome-Wide Association Studies, Ehret GB, Munroe PB et al: Genetic variants in novel pathways influence blood pressure and cardiovascular disease risk. Nature 2011; 478: 103-109.

Supplementary Information accompanies this paper on European Journal of Human Genetics website (http://www.nature.com/ejhg) 